\title{
Revista

\section{NOTA DE INVESTIGACIÓN}

A propósito del libro Sumak Kawsay Yuyay. Antología del pensamiento indigenista ecuatoriano sobre Sumak Kawsay, Antonio Luis Hidalgo-Capitán, Alejandro Guillén García y Nancy Deleg Guazha (eds.), 2014, Huelva y Cuenca, CIM/PYDLOS/FIUCUHU, 367 pp, ISBN 978-84-616-8167-9.

Patricio Cruz y Celis Peniche Escuela en Gestión y Autodesarrollo Indígena Universidad Autónoma de Chiapas patocyc@yahoo.com

El libro de Sumak Kawsay Yuyay que aquí reviso es el producto de la colaboración interinstitucional que desde septiembre de 2013 han forjado la Universidad de Huelva, España, y la Universidad de Cuenca, Ecuador. Semejante intercambio cultural, académico e intelectual se ha centrado en el pensamiento del buen vivir y el desarrollo local sustentable, en un periodo de fortalecimiento histórico del indigenismo latinoamericano. El espíritu general de la obra parece ser resumido en la presentación que los compiladores ofrecen en las primeras páginas, donde describen al sumak kawsay como el «fruto de una curiosa hibridación entre la metáfora de la paja del páramo y el mito del buen salvaje».[18]' Los autores se refieren a aquella hierba que por más que la arranquen no desaparece, y a una noción de existencia social en donde la naturaleza misma brinda toda la armonía necesaria para convivir en paz y prosperidad. Aterrizando la metáfora, vemos que los autores se refieren a los incansables esfuerzos de intelectuales y dirigentes de movimientos sociales indígenas ecuatorianos y bolivianos por hacerse escuchar, así como a una ideología dentro de la cual su cosmovisión particular puede sentirse cómoda y comprendida. El libro en sí es una compilación de diversos artículos publicados entre el 2000 y el 2014 por científicos sociales, figuras públicas e investigadores latinoamericanos enfocados en el pensamiento indígena de los grupos originarios de Bolivia y Ecuador.

A grandes rasgos, todos los textos parten de la premisa central de que el sumak kawsay (entendido como 'vida en armonía' o 'buen vivir') puede ser considerado una alternativa general a los procesos del desarrollo capitalista. En particular, algunos autores consideran que es la mejor apuesta para alcanzar la sustentabilidad socioambiental. Esta interpretación es al mismo tiem-

\footnotetext{
${ }^{1}$ El número entre corchetes es la página del libro comentado.
} 
po la más atractiva y la más controversial en lo que concierne a su recepción fuera de los círculos indígenas de donde es originario el concepto. Por ello, he decidido centrar esta nota en la supuesta relación entre el sumak kawsay y el desarrollo sustentable. A través de una selección de capítulos que hacen particular hincapié en el tema del desarrollo, la sustentabilidad ambiental y la crisis ecológica y social del sistema de producción capitalista, primero revisaré la relación que los propios autores compilados en esta obra trazan entre el sumak kawsay y el desarrollo sustentable. Posteriormente, incluyo un análisis crítico de esta relación que trasciende a los autores revisados, pero que no obstante se adentra en los fundamentos teóricos que sustentan la presente obra. Finalmente, brindo algunas consideraciones en torno al papel de la presente antología para los propósitos de alcanzar un desarrollo sustentable.

En «El concepto del desarrollo sustentable y los pueblos indígenas», Lourdes Tibán comienza por interpretar el desarrollo sustentable «como una forma de conciliar el crecimiento económico (expresado en la concepción del desarrollo) y el equilibrio del ecosistema».[105] Posteriormente, hace una distinción entre las perspectivas indígenas y no indígenas del desarrollo, comenzando a describir estas últimas a partir de los tres discursos de sustentabilidad que Arturo Escobar (1999) define: el discurso liberal, el discurso culturalista y el discurso ecosocialista. El primero de estos es, según Tibán, el más comúnmente aceptado, al ser aquel que originalmente asumió la doctora Bruntland en su famoso informe de 1986. El discurso liberalista parte de la separación de lo económico y lo político, y reduce todo proceso y objetivo de desarrollo a una cuestión económica. El segundo discurso, por su parte, critica la postura liberal y subraya la importancia de la cultura. Asimismo, considera que el crecimiento económico y la conservación de la naturaleza son procesos irreconciliables. Por último, el ecosocialismo que Escobar describe se enfoca en la posibilidad de conseguir una «economía política reformada y centralizada» para los países en desarrollo.[108] Al retomar esta tipología, Tibán asume que el desarrollo sustentable en el discurso no indígena es dominado por una ideología liberal, y «está orientado al crecimiento económico, mas no a la preservación del medio ambiente, el ecosistema, la cultura, la naturaleza, etc.».[109]

A diferencia de la visión economicista del desarrollo sustentable en el discurso no indígena, la aproximación indígena «cree importante considerar las políticas alternativas del desarrollo local y global, para ver si es posible iniciar un desarrollo desde abajo hacia arriba».[109] Tibán prosigue describiendo las políticas 
de desarrollo específicas de tres organizaciones sociales que trabajan de cerca con los pueblos indígenas de Ecuador: la CONAIE, la FENOCIN, y la FEINE. Mientras que la primera propone un «desarrollo integral» que abarque «las dimensiones humanas, naturales, éticas, políticas y culturales de las condiciones de vida de las personas»,[110] la segunda cree en promover un «desarrollo sustentable con identidad», basado en diferentes niveles o sectores socio-políticos que van desde la comunidad hasta la nación. Por último, la FEINE promueve una visión de desarrollo equitativo basado en principios cristianos evangélicos, particularmente subrayando la esfera espiritual del bienestar personal y social, así como la importancia de los valores morales. Tibán concluye que «Todas las propuestas [indígenas] insisten en el respeto a las estrategias tradicionales y formas ancestrales de relación entre el hombre y la naturaleza, que históricamente han sido comprobadas que han dado resultados de protección y conservación del medio ambiente y la seguridad de la vida social».[113]

Inversamente, Silvia Tutillo (2002) hace una defensa similar en favor de la concepción indígena del desarrollo pero a través de criticar las propuestas fallidas de diversas agencias gubernamentales específicas. En «La perspectiva de desarrollo sustentable desde las agencias del desarrollo y la forma de entender el desarrollo de los pueblos indígenas», dicha autora revisa la falta de éxito que han tenido diversos programas de desarrollo, al pretender beneficiar a la población de un país sin considerar la cultura y el pensamiento de su gente. Critica fuertemente proyectos y políticas públicas de este corte, como el modelo de sustitución de importaciones, y el desarrollo local y rural. Específicamente, se enfoca en el fracaso del programa de Desarrollo Rural Integral en Ecuador, así como sanciona nuevamente las pretensiones integradoras de estos modelos. Tutillo concluye que «no es posible hacer propuestas de desarrollo desde las instituciones; hay que lograr comprender las demandas de los pueblos indígenas».[122]

Por su parte, Nina Pacari comienza trazando la distinción que existe entre la visión occidental del sujeto de derecho (enfocada en el individuo) y su visión indígena (enfocado en la colectividad o el grupo). De ahí, Pacari elabora más a fondo sobre el principio de «relacionalidad» que permea toda la coexistencia de los grupos indígenas, y que trasciende al grupo y lo vincula con el medioambiente. En «Naturaleza y territorio desde la mirada de los pueblos indígenas», Pacari describe a partir de numerosos ejemplos la ecología del pensamiento indígena, así como es reflejada por sus concepciones de ser de sí mismos y del medioam- 
biente que lo rodea. La autora defiende la validez práctica y cosmogónica de este pensamiento, abogando por su defensa y su reproducción.

Pablo Dávalos (2008a) arremete contra el neo-liberalismo en «El Sumak Kawsay (Buen Vivir) y las censuras del desarrollo». Particularmente perspicaz y atinada es la manera en que el autor describe a esta doctrina ideológica como si se tratara de un dogma religioso:

En esta noción de crecimiento y desarrollo económico, el discurso neoliberal crea un fetiche al cual rinde tributos, oraciones, y penitencias. El crecimiento económico, según la doctrina neoliberal, resolverá por sí solo los problemas de la pobreza, iniquidad, desempleo, falta de oportunidades, inversión, contaminación y degradación ecológica, etc. El crecimiento económico se convierte en la parusía del capital. En el horizonte utópico hacia el cual necesariamente hay que llegar, a condición de que, obviamente, se dejen libres los mercados y que el Estado respete las reglas de juego del sector privado. En la teología del neoliberalismo, la parusía del crecimiento económico solo puede provenir de la mano invisible de los mercados.[136]

Dávalos pone en evidencia la explotación y la acumulación privada de capital como las bases históricas del sistema capitalista, y reprueba los discursos liberales de progreso y bienestar basados en esta forma de producción socialmente injusta; sobre todo aquellos que hacen parecer que este sistema es incuestionable e inevitable. Concretamente, el autor resume cinco censuras implícitas en el discurso y la práctica neoliberal: la separación del hombre y la naturaleza, la falta de ética en sus principios productivos, la ruptura entre la lógica del capital y la historia y cultura de las sociedades, el olvido de la economía como práctica política por medio de la construcción artificial de la pobreza y, por último, la censura epistémica: aquella que deriva de ignorar o erradicar toda forma de conocimiento que no corresponda con los principios lógicos del sistema de producción capitalista moderno. Ante todo este desorden social e intelectual, el autor propone el sumak kawsay como una — si no la única— alternativa.

En «Reflexiones sobre el Sumak Kawsay (el Buen Vivir) y las teorías del desarro\|l», Dávalos continúa abordando el sumak kawsay como un camino alternativo al bienestar de las personas, haciendo particular hincapié en la destrucción ambiental que ha resultado del sistema hegemónico de producción capitalista: «el Buen Vivir expresa una relación diferente entre los seres humanos y con su entorno social y natural».[150] Mantiene la postura del capítulo anterior, según la cual los pueblos indígenas, sus prácticas tradicionales y su saber ancestral son la solu- 
ción ante los problemas sociales y ambientales que ha acarreado el capitalismo hasta la modernidad. "Llegará un día — profetiza - en el que los conocimientos y saberes ancestrales de los pueblos indígenas sean la única opción para salvar al planeta de la devastación provocada por el libre mercado».[138]

En esta última afirmación está de acuerdo Mónica Chuji en «Modernidad, desarrollo, interculturalidad y Sumak Kawsay o Buen Vivir» (2009) y en «Sumak Kawsay versus desarrollo» (2010). De hecho, la autora coincide plenamente con la postura de Dávalos al ver la ideología del nuevo capitalismo como perversa, y considerar el sumak kawsay «alternativa al progreso, al desarrollo, a la modernidad»;[157] el camino hacia el mismo siendo la interculturalidad. Hay que resaltar que los textos de Chuji aportan mucho a esclarecer la defensa del sumak kawsay como alternativa, a través de una descripción somera de sus diferencias particulares con el desarrollo:

El Sumak Kawsay es un concepto muy atávico, creado a través de la práctica y la experiencia de vida de los pueblos indígenas. El sumak kawsay no es un producto, no es cuantificable, no es acumulable, no tiene un valor monetario; al contrario, es un estado del ser y estar, de manera individual y colectiva, y de su entorno, en el mundo [...] En este contexto, el Sumak Kawsay se diferencia del concepto de «desarrollo» porque no contempla, como prioridad número uno y como principio y fin, la acumulación monetaria a partir de la exploración indiscriminada de la naturaleza.[232-233]

¿Y cuáles serían los objetivos del sumak kawsay? Pues hay una gran cantidad de textos en la obra que aquí reviso que ahondan detalladamente en el tema. Más allá de su definición como buen vivir, podemos entenderlo — particularmente bajo su interpretación como alternativa al desarrollo y como discurso orientado al desarrollo sustentable- como un principio de armonía: entre seres humanos y entre el hombre y el medio ambiente. Silvia Tutillo afirma que «la relación y el manejo del medio ambiente por parte de los pueblos indígenas se dan a partir de sus usos y costumbres, que se encuentran plasmados en su cosmovisión».[122] Según Luis Macas, otro de los compilados en el libro, esta cosmovisión está conformada por prácticas de organización comunitaria y principios de reciprocidad, integralidad, complementariedad y relacionalidad; principios que, según los autores revisados, difieren radicalmente del individualismo, la mercantilización y la homogenización cultural característicos del pensamiento y los principios capitalistas. 


\section{LA NOCIÓN DEL SUMAK KAWSAYY EL DESARROLLO SUSTENTABLE: UNA REVISIÓN CRÍTICA}

Tal parece que los defensores del sumak kawsay confían en la posibilidad de que todo el mundo pueda incorporar esta noción ancestral en su estilo de vida $-\mathrm{y}$ de hecho tenga que hacerlo si quiere alcanzar la sustentabilidad y el equilibrio socio-ambiental_. Ahora bien, que el concepto sea aparentemente incomparable e incompatible con el de desarrollo tiene algunas implicaciones que problematizan su entendimiento y aplicación fuera de los círculos indígenas desde donde surge. A grandes rasgos, podemos mencionar tres ejes temáticos principales que intersecan el discurso de los defensores del sumak kawsay aquí revisados, en los que pudiera surgir un conflicto al querer perseguir el desarrollo sustentable: el sujeto del desarrollo, el concepto de desarrollo y la sustentabilidad del desarrollo.

En un primer caso, la generalización entre lo indígena y lo no indígena que Lourdes Tibán hace exacerba la percepción de barreras étnicas insuperables, sobre todo cuando la etnia misma es entendida esencialmente. El problema es más grave cuando la compleja realidad de los procesos contemporáneos demuestra que asumir necesariamente un buen vivir a partir de la adscripción a una etnia indígena no es siempre una apuesta certera. De igual manera resulta erróneo generalizar a todos los grupos indígenas dentro de un mismo sentir social y político —ni siquiera acerca de los medios por los cuales se alcanzarían dichos objetivos-. Por ejemplo, existen grupos indígenas que jamás contemplarían al Estado dentro de sus estrategias de acción política, ya que consideran ilegítima la participación de este en la realización de su bienestar. En este caso estaríamos hablando de países en que los grupos indígenas no cuentan con mayor representación política, ni son respaldadas las políticas públicas de desarrollo por una legislación pluriétnica.

Uno puede dudar qué tan inclusivo es el discurso del sumak kawsay, cuando discursos no indígenas se muestran — por lo menos en papel_ mucho más dispuestos a considerar a la diversidad de actores sociales del planeta. Por ejemplo, el desarrollo sustentable en su acepción fuerte no solo considera la sustentabilidad de los procesos de desarrollo a largo plazo y para las futuras generaciones (sustentabilidad temporal), sino también el acceso universal a los medios de desarrollo (sustentabilidad espacial) (Minguez y Asencio 2008). De igual manera, la Agenda 21 se muestra explícita en torno a la importancia del papel que pudieran 
desempeñar las poblaciones originarias para alcanzar el desarrollo sustentable (ONU 1992, capítulo 26). Hay que reconocer que la tan criticada tradición liberal -en la cual se insertan las acciones y los discursos de los organismos supranacionales como la ONU - demuestran ser más incluyentes y universalistas en torno al sujeto del desarrollo, por lo menos en teoría. Esto es cierto, aun cuando la adopción de un discurso de sustentabilidad débil y la subordinación de los objetivos del programa al mercado neoliberal parecieran descalificar esta afirmación (Lorek y Fuchs 2013). En cualquier caso, la generalización de lo indígena y lo no indígena pareciera equivocada, aún más si consideramos la cantidad abrumadora de críticas al desarrollo y propuestas de sustentabilidad alternativas (Sachs 1992, Esteva 1996, Martínez-Allier 1992 y 2009, Leff 2004, Foladori y Pierri 2005, Toledo 1993 y 2008, Latouche 2009, Gudynas 2004 y 2011) que provienen de un sector que no se reconoce como indígena. El encasillamiento de lo no indígena dentro de un supuesto discurso economicista impediría que las virtudes de estas propuestas fueran del todo consideradas crítica y objetivamente, sino descartadas a priori con base en criterios ideológicos.

En un segundo caso, parece acelerada o prematura la conceptualización que los defensores del buen vivir hacen del desarrollo capitalista y el crecimiento económico, descalificándolos como si fueran «solo un mito». Ante el grado de complejidad que continúa asumiendo el sistema de producción capitalista, resulta necesario reconocer que este no ha sido del todo arbitrario, casual, ni necesariamente impuesto. Ya sea como víctimas o victimarios, algo en el sistema capitalista apela a las personas de todo el mundo. Para darnos una idea de cuáles pudieran ser estas ventajas, podríamos echar un vistazo a Goklany (2007). ${ }^{2}$ El libro, en gran medida una recopilación y representación gráfica de información estadística internacional, demuestra que las vidas humanas de todo el mundo se han beneficiado por el modelo de desarrollo capitalista, por lo menos en lo que concierne al aumento en la expectativa de vida, la nutrición, la alfabetización, y la escolaridad básica, así como la reducción en las tasas de mortalidad infantil, maternas, el trabajo infantil, entre otras. Ciertamente ni este ni ningún otro libro podrá negar la abrumadora devastación ambiental a cuyo costo se han alcanzado estos beneficios, ni lo preocupantemente rápida que comienza a ser la desigualdad social en países de todo el mundo (Milanovic 2010). No obstante

${ }^{2}$ El título se traduce al español como El estado mejorado del mundo: por qué vivimos vidas más longevas, saludables y cómodas en un planeta más limpio. Ciertamente, la última parte de esta afirmación está sujeta a debate. 
su falta de sustentabilidad ambiental y social, lo importante es no subestimar al desarrollo capitalista, sobre todo porque este involucra cada vez más y más exitosamente al sector indígena.

En efecto, pareciera que los mismos grupos indígenas hubieran asumido como estrategia de supervivencia y fortalecimiento cultural las relaciones económicas con el mercado (incluso el mercado internacional y global), en tanto que una mejor situación económica les permite continuar reproduciendo, hasta cierto punto, sus prácticas y valores tradicionales; y esto es igualmente cierto para aquellos grupos indígenas organizados a nivel comunitario, regional o nacional (Zárate 2002). Como bien dice un autor:

Los indios no son más un factor marginal en la economía de los países. Probablemente no hacen aportes significativos a las cuentas nacionales y su contribución real a la producción de alimentos no es muy importante; sin embargo como un resultado de la desarticulación de las economías de subsistencia y del desarrollo en general, se han incorporado plenamente al mercado interno bajo todas las modalidades posibles de inserción; y en el contexto de una creciente informalización, lejos de asumir formas ortodoxas tienden a conservar, reproducir, y fortalecer maneras culturales particulares; que les permiten construir estrategias de sobrevivencia en las cuales el ejercicio de su identidad como pueblos resulta fundamental (Iturralde 1991:35-36).

Esta inserción económica y transformación del indio en sujeto económicamente involucrado es cierta incluso para los pueblos aymaras y quechuas (Ticona 2011). Puesto que lo recién expuesto para los pueblos indígenas es doblemente cierto a nivel individual, cualquier esencialismo — similar al que se suscribe Nina Pacari - debe ser superado por la posibilidad más realista de que el indígena también es un sujeto con identidad dinámica (Chávez 2003, Balslev 2008), libre de formularse sus propios intereses personales (Van de Berghe 1981). Los individuos indígenas pueden elegir reproducir nuevas formas de vida sin necesariamente caer en una aculturación, al decidir qué elementos culturales e identitarios conservar o incorporar. De ahí que las identidades indígenas en la modernidad

${ }^{3}$ El hecho de ser pueblo originario o ancestral no está supeditado a la voluntad personal o espontánea de querer ser kichwa, shwar, aymara o kolla, sino que quien pertenece a un pueblo indígena nace, vive y muere con esa identidad asumida desde su conciencia y aceptada por los demás como parte integrante de su caminar histórico-cultural e identitario.[129] 
puedan comprenderse mejor a partir de una noción de continuidades y no necesariamente de rupturas (Renard 2004).

Por el otro lado, también debemos ser confrontados con la cruda realidad de que los sujetos indígenas decidan voluntariamente dejar de reproducir sus elementos culturales del todo - sin ser producto de una imposición o un etnocidio-. Esto es ocasionalmente visible en aquellas personas que conscientemente deciden migrar a un medio urbano, no por la falta de sostenibilidad de sus medios rurales, sino a partir de una serie de beneficios económicos, políticos y sociales que perciben de la ciudad (Rubio et al. 2000). Nuevamente, estas decisiones están respaldadas por un cálculo consciente de costos y beneficios, y no necesariamente por una imposición violenta. Como lo demuestra la siguiente cita respecto de la sustitución lingüística:

Un estudiante navajo me expuso una vez el problema con bastante crudeza: «si tengo opción entre vivir en un hogar a una milla del agua más cercana, donde mi hijo crecerá hablando navajo, y mudarme a una casa en la ciudad con tuberías donde hablará inglés con sus vecinos, elegiré inglés y un cuarto de baño» (Spolsky 1989:451). ${ }^{4}$

Esto desmiente una vez más la afirmación apresurada de que «el crecimiento económico [...] [únicamente] es obra de los mercados y, a su vez, de las empresas privadas [...] en su forma más moderna: la corporación».[135] Los beneficios percibidos en la cita de más arriba son la causa y a su vez la consecuencia del éxito del mercado para integrar a diversos actores socioculturales a sus filas de productividad y consumo. Por si fuera poco, esta afirmación se ve respaldada por una serie de discursos y movimientos que nos son familiares, como el desarrollo endógeno o local, o incluso el mismo etnodesarrollo, los cuales indirectamente fomentan la integración del indígena al sistema capitalista a partir de un discurso centrado en la cultura local y la organización social (Tejera 2006). Esto nos lleva a preguntarnos qué tanto difiere el discurso culturalista del sumak kawsay frente a estos últimos.

La pregunta anterior es un tanto difícil de responder ya que hay una tendencia a equiparar al desarrollo (en general) con el capitalismo y su modelo de crecimiento económico (en particular), lo que hace muy difícil vislumbrar en la práctica la propuesta del sumak kawsay. Puesto de esta manera, resulta más aparente que el sumak kawsay no es un modelo de desarrollo en sí, sino un producto ideoló-

${ }^{4}$ Citado en Baker y García (1997:83). 
gico de la cosmovisión de ciertos grupos indígenas. Lo que quiere decir que, dependiendo de la interpretación que se le dé, de ello podrán generarse prácticas ahora más o menos sustentables, ahora más o menos capitalistas. Tampoco ayuda al escepticismo al que aquí me suscribo que el discurso del sumak kawsay lo afirme no solo como una alternativa al desarrollo capitalista, sino al desarrollo en general. La equiparación del desarrollo con el capitalismo como sinónimos, o a lo sumo como conceptos indisociables, es respaldada por un experto del desarrollo como Wolfgang Sachs (1997) en un artículo titulado «No hay sustentabilidad sin desarrollo». En este sentido, Sachs mismo reduce el desarrollo a un discurso, "una forma de pensamiento». No obstante estas posturas, considero que ni los defensores del desarrollo ni sus críticos están dispuestos a considerar la posibilidad de definir al desarrollo en términos concretos. Libre de alguna ideología, esta interpretación al desarrollo humano haría referencia a los procesos biológicos y ecológicos por medio de los cuales una especie como la nuestra permanece a flote (Richerson et al. 1996); tarea que sin embargo ha conducido a la generación de problemas de desarrollo más grandes y costosos de resolver, en el momento de abordar otros tantos originalmente más sencillos (Tainter 1990).

Esto nos lleva a un último punto que es el entendimiento que puedan tener los defensores del sumak kawsay de la sustentabilidad en sí, junto con sus implicaciones, sus criterios lógico-científicos, y los métodos y los mecanismos evaluadores. Resulta muy difícil invalidar la afirmación de que las prácticas de subsistencia de los grupos indígenas son, de hecho, más sustentables y ecológicas que aquellas que promueven los más modernos procesos de industrialización, urbanización, especialización laboral y destrucción creativa tecnológica. Sin embargo, no siempre es fácil concluir qué tanto es esta supuesta superioridad ecológica un producto históricamente contingente (Diamond 1997) y qué tanto un producto de la cosmovisión particular de los grupos indígenas (Rappaport 1968). Según la primera de estas explicaciones, distintos procesos históricos hubieran podido haber llevado a las sociedades amerindias a desarrollar tecnologías similares que las euroasiáticas, con sus respectivas consecuencias socioambientales. Inversamente, esta perspectiva afirma que fueron muchas las sociedades y culturas euroasiáticas que llegaron a desarrollarse bajo niveles de sostenibilidad ambiental similares a los de algunos pueblos indígenas americanos actuales. Esto querría decir que la «sustentabilidad» en realidad radica en la influencia de ciertos condicionamientos históricos sobre el estilo de vida de las personas, y no en su adscripción étnico-identitaria. 
Pero supongamos por un momento que concedemos la generalización de lo indígena, y reconocemos que la superioridad ecológica de los estilos de vida particulares de los grupos indígenas puede ser objetivamente demostrada como el producto de su cosmovisión. Aun así existen amplias posibilidades de que esta sabiduría fuera el producto de años de adaptación cultural a un ambiente determinado (Richerson y Boyd 2005). De ser así, es muy probable que la velocidad y magnitud de los actuales cambios ambientales — históricamente sin precedentes- no garantizarían que tales estrategias y métodos de supervivencia —reproducidos intergeneracionalmente- sigan siendo válidas o puedan efectivamente hacer frente a las exigencias del cambio climático, o a los consecuentes problemas sociales. ${ }^{5}$ Sólo un pensamiento antiecológico y etnocéntrico podría afirmar la posibilidad de que un pequeño grupo poblacional pueda permanecer inmune a los desbalances socioambientales a los que el resto del mundo está expuesto. Afortunadamente, los principios de relacionalidad del sumak kawsay afirman que «no se puede vivir bien si los demás viven mal» (Giraldo 2014:16). Aun así, mi escepticismo parece verse reforzado cuando, por un lado, se percibe la cercana dependencia de las prácticas de subsistencia de los grupos indígenas a un medio ambiente equilibrado y saludable, así como por la poca importancia que conceden los defensores del sumak kawsay a los procesos productivos subyacentes, y la importancia que adquieren como sustento físico-material de toda su cosmovisión (Harris 1979). En este caso, estaríamos ante una mala interpretación de una economía de subsistencia considerada ideológica y no productivamente excepcional.

Para acotar el vacío que existe entre las concepciones indígenas y no indígenas del desarrollo y la sustentabilidad se ha sugerido un diálogo de saberes (Pérez y Argueta 2011, Leff 2011). Sin embargo, esta posibilidad también tiene sus dificultades, por lo menos cuando la propuesta es desarrollada dentro de un multiculturalismo político al que se suscriben la mayoría de los defensores de la obra que aquí reviso. Un primer problema es epistemológico, y tiene que ver con la falta de consenso entre las nociones de verdad, realidad y, sobre todo, ser humano en sí. Existe una tendencia intelectual por parte de las ciencias sociales a interpretar el conocimiento percibido de los grupos indígenas en términos relativistas, lingüísticamente relativistas, constructivistas, y a hacerlo fenomenológi-

${ }^{5}$ El ritmo de crecimiento del capitalismo acota las posibilidades de supervivencia de la especie humana, en un debate que ahora cobra un sentido y una urgencia reales: de continuar con el actual ritmo de producción y consumo, las teorías del calentamiento global predicen una catástrofe ecológica de consecuencias inimaginables.[149] 
ca, interpretativa e ideográficamente. Al contrario, los postulados científicos de, por ejemplo, las ciencias ambientales, las ciencias naturales y una minoría de las ciencias sociales adoptan una perspectiva objetivista y realista, basados en una metodología nomotética y materialista (Ingold 2008). De esta forma, mientras que los primeros analizan y describen al ser humano en sociedad a partir de la ideología de su tiempo, los procesos simbólicos que rigen esas ideologías y los determinantes históricos (Giraldo 2014), las segundas describen al hombre como un organismo sujeto a leyes y procesos biológicos, físicos, socioculturales, etcétera. Algunos autores consideran que la falta de coherencia entre las concepciones indígenas del universo y las del pensamiento científico se deben meramente a una desigualdad social, y no a profundas diferencias epistemológicas (Hersch 2011). Sin embargo, el hecho de que estas diferencias sólidamente persistan en el mundo académico (no indígena) debería bastar para considerar la posibilidad de que quizá sean irreconciliables después de todo (Padrón 2007).

Un segundo problema se desprende directamente de este, y tiene que ver con que, hasta este punto en la evolución del pensamiento científico, la única conclusión que prácticamente todas las disciplinas científicas comparten es que el mundo natural, incluyendo al hombre, es algo complejo, no siempre intuible, y difícil de conocer e interpretar (Pinker 2002). Esto no necesariamente significa que las intuiciones de los pueblos indígenas acerca de su entorno natural local no puedan ser hasta ahora mayormente acertadas. Sencillamente, la conciliación de los procesos del desarrollo (sociales, ambientales, culturales, físicos, biológicos, tecnológicos, etc.) con la posibilidad de hacerlos sustentables (a largo plazo y a través de las poblaciones del planeta) involucra o directamente exige un grado de conocimiento que no es meramente intuible, ni biológica ni culturalmente. ${ }^{6}$ De manera que si — por alguna razón - uno considera necesario un conocimiento científicamente profundo del mundo natural para definir los criterios de sustentabilidad del desarrollo humano en el contexto actual, se tendrán

\footnotetext{
${ }^{6}$ Como apunta Steven Pinker: «Estas formas [de sentido común] de conocimiento y las intuiciones profundas son útiles para los estilos de vida de pequeñas sociedades analfabetas y sin estado, que viven de la tierra, sobreviven por medio de sus habilidades y dependen de aquello que puedan cargar consigo mismas. [...] Para muchos otros campos de conocimiento, la mente no pudo haber evolucionado una maquinaria específica, el cerebro y el genoma no muestran señal de especialización en ellos, y las personas no demuestran ningún entendimiento intuitivo espontáneo ya sea en la infancia o posteriormente. Estos incluyen a la física moderna, la cosmología, la genética, la evolución, la neurociencia, la embriología, la economía y las matemáticas. No es sólo que tengamos que ir a la escuela o leer libros para aprender estos temas. Es que no tenemos las herramientas mentales para comprenderlos intuitivamente» (Pinker 2002:221) [la traducción es mía].
} 
que superar algunas barreras ideológicas para poder alcanzar el buen vivir que el sumak kawsay describe.

Una última dificultad tiene que ver con que, políticamente hablando, no existe un terreno o contexto en el que esta superación (o suspensión) ideológica pueda llevarse a cabo. El programa político multiculturalista (dentro del cual se han promovido las constituciones pluriétnicas de Bolivia y Ecuador) rechaza la posibilidad de que tenga que desarrollarse un solo terreno político intermedio, dentro del cual todas las diferentes interpretaciones del universo (cosmovisiones) sean juzgadas e interpretadas a partir de un solo y mismo punto de referencia. Esto ha derivado en serias críticas para el multiculturalismo, el cual es interpretado como inconducente a la realización del bienestar humano y la justicia social (Nussbaum 1999), y como conducente a la violencia interétnica (Sen 1999), el racismo, el etnocentrismo, e incluso al capitalismo (Zizek y Jameson 1998). Por ello autores como Martha Nussbaum y Amartya Sen consideran importante ir en defensa de los valores universales, y colocar la razón sobre la identidad. No es necesario defender la república democrática y liberal como el único esquema político a seguir para reconocer la importancia de que cualquier modelo político reconozca principios éticos universales como la libertad del individuo ante la sociedad o la defensa de los derechos humanos (Ignatieff 2001). Quizá existan buenas razones para considerar los valores universales y la objetividad de la razón como herramientas mayormente útiles para la comprensión y cooperación entre seres humanos provenientes de diversos contextos socioculturales; más allá de su satanización como supuestas herramientas discursivas al servicio de una ideología política o doctrina económica específica (exclusivamente conveniente a los intereses del mercado, la clase burguesa, o los países imperialistas [Santos 2014]). En este sentido, un cosmopolitanismo político (Appiah 2007) estaría mejor preparado que el multiculturalismo para llevar a cabo la tarea de sintetizar o mediar el universo de diversidad cultural e ideológica bajo un solo marco lógico de verdad y de respeto entre humanos, en el cual sería posible la persecución conjunta de un objetivo de magnitudes globales como el de la sustentabilidad del desarrollo.

\section{CONCLUSIÓN}

A raíz de mi revisión del libro Sumak Kawsay Yuyay, pareciera que el concepto de sumak kawsay no necesariamente conlleva un desarrollo sustentable que sea universal y pluriidentitario. Aun así, existe la posibilidad de que el concepto 
pueda ser mayormente útil para las premisas de un etnodesarrollo o desarrollo autónomo indígena, aunque no sin sus respectivas dificultades y contradicciones. La operatividad del sumak kawsay podría darse en la práctica de dos formas distintas. La primera de ellas niega el desarrollo por completo. La manifestación explícita de la gran incompatibilidad que existe entre el sumak kawsay y el desarrollo, así como las profundas diferencias epistemológicas entre uno y otro, impiden hacer más inteligible el concepto fuera de los grupos indígenas. Esto hace parecer al buen vivir como exclusiva o mayormente congruente con aquella interpretación de la realidad que contemplan sus creadores. Esto se traduciría a un buen vivir que fuera puesto en práctica únicamente por y para aquellas personas que se identifican con el concepto y lo entienden cabalmente, acotando su operatividad a los círculos indígenas de donde proviene el concepto. Otra posibilidad radica en la incorporación del sumak kawsay a los discursos del desarrollo como una propuesta más de desarrollo alternativo, como el «desarrollo con identidad».? Sin embargo, tras reconocer la grandísima incompatibilidad entre el sumak kawsay y el desarrollo, perseguir un terreno intermedio potencialmente arriesgaría reproducir aquellas acciones que los críticos del multiculturalismo reconocen como una integración al sistema capitalista que sea «culturalmente amigable». Por ejemplo, agencias de desarrollo como el Banco Interamericano de Desarrollo no han dudado en hacer uso de una retórica similar. Deruyttere (2001) subraya la misma sabiduría ecológica de los pueblos indígenas que otrora hemos cuestionado como romántica, para luego sugerir las aportaciones que esa misma racionalidad particular pudiera tener para una economía intercultural. No obstante la aparente inocuidad del modelo intercultural de Deruyttere, resulta fácil trazar una línea entre su propuesta de dejar de ver a los indígenas como obstáculos para el desarrollo y los intereses explícitos del Banco Interamericano del Desarrollo por integrar comercialmente a las poblaciones de América Latina y el Caribe a un sistema capitalista que algunos consideramos inherentemente insustentable. Resulta interesante que solo uno de los autores que figuran en la antología que aquí reviso, Atawallpa Oviedo, subraya de manera explícita las

\footnotetext{
${ }^{7}$ Por ejemplo, Lourdes Tibán al describir el concepto señala sus implicaciones políticas: «El desarrollo con identidad implica que el desarrollo no sea ajeno a la comunidad, que esté vinculado a la realidad comunitaria, que no altere la concepción de la tierra que tiene el indígena, el campesino y el negro, y que las particularidades, en general, sean consideradas en un desarrollo participativo y de base. Sin embargo, es de resaltar que en la propuesta analizada no se pueden establecer las formas de articulación entre estos diferentes niveles; en efecto, ¿cuáles serían las formas de articulación de la comunidad o de las bases con el resto del país?».[111]
} 
inmensas dificultades para articular un desarrollo con identidad o un desarrollo sustentable con los objetivos y principios fundamentales del sumak kawsay. Su crítica me parece resume concretamente las dificultades que aquí he venido describiendo y analizando:

Todos estos organismos deben estar muy agradecidos con la izquierda socialista e indigenista, cómplice del famoso cuento del desarrollo, de la integración, del progreso, de la inclusión, de la modernidad, que surgió con las tendencias neoliberales del crecimiento económico, pero que fue fácilmente adoptado también por la izquierda, solo cambiándole cada vez más de apellidos, pero siendo al final lo mismo: desarrollo sustentable, desarrollo sostenible, desarrollo limpio, desarrollo comunitario, desarrollo con identidad [...] No necesitaron nuevas conquistas, nuevas formas de colonialismo beligerante, simplemente necesitaron de una izquierda idealista y de una dirigencia indígena civilizada y desarrollada, para que el sistema económico capitalista penetre y se consolide fácilmente en los pueblos y las comunidades.[313]

En tanto que no se llegue a un acuerdo en torno a cuál es la definición objetiva y universalmente válida de los conceptos centrales al desarrollo sustentable (como lo son los sujetos del desarrollo, el desarrollo, la sustentabilidad misma, y los indicadores particulares para alcanzarla) la posibilidad de una cooperación consensuada entre los grupos indígenas y los individuos no indígenas para alcanzar el desarrollo sustentable parece una tarea difícil y compleja. Siendo este el estado de las cosas, considero improbable la afirmación que Dávalos [138] hace al considerar el sumak kawsay como la «única opción» para alcanzar la sustentabilidad del desarrollo. No obstante, la antología que aquí he revisado críticamente ofrece al público un terreno fértil para la discusión, al estar compuesta por una gran diversidad de voces y opiniones, cada una de las cuales imagina el sumak kawsay de maneras ligeramente distintas, pero siempre en un explícito rechazo y rebeldía ante el desarrollo capitalista que actualmente impera alrededor del mundo. Por ello, sea el sumak kawsay una alternativa real o imaginada a los problemas de sustentabilidad con los cuales nos confronta el desarrollo, la riqueza de su propuesta radica precisamente en ser articulada desde las profundidades del corazón indigenista ecuatoriano. 


\section{FUENTES DE CONSULTA}

Appiah, Kwame A., 2007, Cosmopolitanism: ethics in a world of strangers, Nueva York, W.W. Norton \& Company.

Balslev, Helene, 2008, «La etnicidad de los mazahuas en un municipio mexiquense, un estudio de violencia simbólica», en Daniel Gutierrez y Helene Balslev (coords.), Revisitar la etnicidad, miradas cruzadas en torno a la diversidad, México, El Colegio de Sonora/El Colegio Mexiquense/Siglo XXI Editores, pp. 249-273.

Berghe, Pierre van den, 1981, The ethnic phenomenon, Nueva York, Elsevier.

Chávez, Ma. Eugenia, 2003, Identidades y cambios culturales, los mazahuas de San Antonio, Pueblo Nuevo, México, Universidad Autónoma de Chapingo.

Deruyttere, Anne, 2001, Pueblos Indígenas, globalización y desarrollo con identidad: algunas reflexiones de estrategia, Washington, Banco Interamericano de Desarrollo.

Diamond, Jared, 1997, Guns, Germs \& Steel, the fates of human societies, Nueva York, W.W. Norton \& Company.

Escobar, Arturo, 1999, «El desarrollo sustentable: diálogo de discursos», en Arturo Escobar (comp.), El final del salvaje, naturaleza, cultura y política en la antropología contemporánea, Santa Fe de Bogotá, CEREC/ICAN, pp. 75-97.

Esteva, Gustavo, 1996, "Desarrollo», en Wolfgang Sachs (ed.), Diccionario del desarrollo y subdesarrollo: Una guía del conocimiento como poder, Lima, PRATEC.

Foladori, Guillermo y Pierri Naína (coords.), 2005, ¿Sustentabilidad? Desacuerdos sobre desarrollo sustentable, México, H. Cámara de Diputados / Universidad de Zacatecas / Porrúa.

Giraldo, Omar F., 2014, Utopías en la era de la supervivencia, una interpretación del buen vivir, México, Ítaca / Universidad Autónoma Chapingo.

Goklany, Indur, 2007, The Improving State of the World: Why We're Living Longer, Healthier, More Comfortable Lives on a Cleaner Planet, Washington, The Cato Institute.

Gudynas, Eduardo, 2004, Ecología, economía y ética del desarrollo sostenible, Uruguay, CLAES.

2011, «Debates sobre el desarrollo y sus alternativas en América Latina: una breve guía heterodoxa», en M. Lang y D. Mokrani (eds.), Más allá del desarrollo, Quito, Grupo Permanente de Trabajo sobre Alternativas al Desarrollo / Fundación Rosa Luxemburgo / AbyaYala, pp. 21-53.

Harris, Marvin, 1979, Cultural Materialism, the struggle for a science of culture, EUA, Alta Mira Press.

Hersch, Paul, 2011, «Diálogo de saberes: ¿para qué? ¿para quién? Algunas experiencias desde el programa de investigación Actores Sociales de la Flora Medicinal en México, del Instituto Nacional de Antropología e Historia», en Arturo Argueta, Eduardo Corona y Paul Hersch (coords.), Saberes colectivos y diálogo de saberes en México, Cuernavaca, CRIM-UNAM/Universidad Iberoamericana, pp. 173-200.

Ignatieff, Michael G., 2001, Human Rights as Politics and Idolatry, Canadá, Anansi Press. Ingold, Tim, 2008, "Anthropology is not Ethnography», Proceedings of the British Academy, 154, pp. 69-92. 
Iturralde, Diego A., 1991, «Los pueblos indios como nuevos sujetos sociales en los Estados latinoamericanos», Nueva Antropología, 11(39), pp. 27-40.

Latouche, Sergei, 2009, Decrecimiento y posdesarrollo: el pensamiento creativo contra la economía del absurdo, España, Icaria.

Leff, Enrique, 2004, Racionalidad ambiental: la reapropiación social de la naturaleza, México, Siglo XXI Editores.

, 2011, «Diálogo de saberes, saberes locales y racionalidad ambiental en la construcción social de la sustentabilidad» en Arturo Argueta, Eduardo Corona y Paul Hersch (coords.), Saberes colectivos y diálogo de saberes en México, Cuernavaca, CRIM-UNAM / Universidad Iberoamericana, pp. 379-391.

Lorek, Sylvia y Doris Fuchs, 2013, «Strong Sustainable Consumption Governance - precondition for a degrowth path?» Journal of Cleaner Production, 38, pp. 36-43.

Martinez-Allier, Joan, 2009, El ecologismo de los pobres: conflictos ambientales y lenguajes de valores, Barcelona, Icaria.

, 1992, De la economía ecológica al ecologismo popular, Barcelona, Icaria.

Milanovic, Branko, 2011, Los que tienen y los que no: una breve y singular historia de la desigualdad global, España, Alianza.

Mínguez Moreno, Olga y María José Asencio Coto, 2008, Sustainability of Territorial Projects: a proposal, Huelva, España, International Conference of Territorial Intelligence.

Nussbaum, Martha, 1999, In Defense of Universal Values, Indiana, University of Notre Dame, The Joan B. Kroc Institute for International Peace Studies.

Organización de las Naciones Unidas (ONU), 1992, Agenda 21, Conferencia de las Naciones Unidas sobre el Medio Ambiente y el Desarrollo, Río de Janeiro, junio.

Padrón, José, 2007, «Tendencias epistemológicas de la investigación científica en el siglo XXI», en Cinta de Moebio, Revista de Epistemología de Ciencias Sociales, Chile, Universidad de Chile, 28, pp. 1-28.

Pérez Ruiz, Maya Lorena y Arturo Argueta Villamar, 2011, "Saberes indígenas y diálogo intercultural», Cultura y representaciones sociales, año V, 10, pp. 31-56.

Pinker, Steven, 2002, The Blank Slate: the modern denial of human nature, EUA, Penguin. Rappaport, Roy, 1968, Pigs for the ancestors, New Haven, Yale University Press.

Renard, Ma. Cristina, 2004, «Tradición y ruptura en Tzo'Ontahal, un pueblo en los Altos de Chiapas», en Eduardo Sandoval y Manuel Baeza (coords.), Cuestión étnica, culturas, construcción de identidades, México, UNAM-Caracol.

Richerson, Peter y Robert Boyd, 2005, The Origin and Evolution of Cultures, Oxford, Oxford University Press.

Richerson, Peter, Monique Borgerhoff y Bryan J. Vila, 1996, Principles of Human Ecology, EUA, Pearson Custom Publishing.

Rubio, Miguel Ángel, Saúl Millán y Javier Gutiérrez (coords.), 2000, La migración indígena en México, México, Estado del Desarrollo Económico y Social de los Pueblos Indígenas de México, Instituto Nacional Indigenista y Programa de las Naciones Unidas para el Desarrollo.

Sachs, Wolfgang (ed.), 1992, The Development Dictionary: a guide to knowledge as power, Reino Unido, Zed Books. 
Sachs, Wolfgang, 1995, "No Sustainability Without Development», Critical Reflections of the Culture of the West, Ocassional Paper 1, Bruselas, The Ecumenical Association for Church and Society.

Santos, Boaventura de Sousa, 2014, Epistemologías del sur, México, Ediciones Akal.

Sen, Amartya, 1998, Reason Before Identity, Oxford, The Romanes Lectures for 1998, Oxford University Press.

Spolsky, Bernard, 1989, Conditions for Second Language Learning, introduction to a general theory, Oxford, Oxford University Press.

Tainter, Joseph, 1990, The Collapse of Complex Societies, Cambridge, Cambridge University Press.

Tejera, Pedro, 2006, "Objeciones a la teoría del desarrollo local (desde una perspectiva tercermundista)», III Conferencia Carlos Marx y los desafíos del siglo XXI, La Habana, mayo.

Toledo, Victor M., 1993, "La racionalidad ecologica de la produccion campesina», en Eduardo Sevilla Guzman y Manuel Gonzalez de Molina Navarro (eds.), Ecología, campesinado e historia, España, La Piqueta.

,2011, "La Constitución del "indio" boliviano y su condición actual: el concepto y lo que significa», $6^{\circ}$ Congreso de la Asociación de Estudios Bolivianos, Sucre, 2630 de junio.

Toledo, Victor M., 2008, «Metabolismos rurales: hacia una teoría económico-ecológica de la apropiación de la naturaleza», Revista Iberoamericana de Economía Ecológica, 7, pp. 1-26.

Zárate Hernández, J. Eduardo, 2002, "Ciudadanía, comunidad y modernidades étnicas», en Marco Calderón, Willem Assies y Ton Salman (eds.), Ciudadanía, cultura política y reforma del Estado en América Latina, México, El Colegio de Michoacán / Instituto Federal Electoral de Michoacán.

Žižek, Slavoj y Fredric Jameson, 1998, Estudios culturales y reflexiones sobre el multiculturalismo, México, Paidós. 